\title{
Analysis of Bell-Type Experiments and Its Local Realism
}

\author{
Koji Nagata ${ }^{1}$, Tadao Nakamura ${ }^{2}$ \\ ${ }^{1}$ Department of Physics, Korea Advanced Institute of Science and Technology, Daejeon, Korea \\ ${ }^{2}$ Department of Information and Computer Science, Keio University, Yokohama, Japan \\ Email: ko mi na@yahoo.co.jp, nakamura@pipelining.jp
}

Received 16 June 2015; accepted 17 July 2015; published 20 July 2015

Copyright (C) 2015 by authors and Scientific Research Publishing Inc.

This work is licensed under the Creative Commons Attribution International License (CC BY). http://creativecommons.org/licenses/by/4.0/

(c) (i) Open Access

\section{Abstract}

We investigate the violation factor of the original Bell-Mermin inequality. Until now, we have used an assumption that the results of measurement are \pm 1 . In this case, the maximum violation factor is as follows: $2^{(n-2) / 2}(n=$ even $)$ and $2^{(n-1) / 2}(n=$ odd $)$. The quantum predictions by $n$-partite Greenberger-Horne-Zeilinger state violate the Bell-Mermin inequality by an amount that grows exponentially with $n$. Recently, a new measurement theory is proposed [K. Nagata and T. Nakamura, International Journal of Theoretical Physics, 49, 162 (2010)]. The values of measurement outcome are $\pm 1 / \sqrt{2}$. Here we use the new measurement theory. We consider a multipartite GHZ state. We use the original Bell-Mermin inequality. It turns out that the original Bell-Mermin inequality is satisfied irrespective of the number of particles. In this case, the maximum violation factor is as follows: $1 / 2(n=$ even $)$ and $1 / \sqrt{2}(n=$ odd $)$. Thus the original Bell-Mermin inequality is satisfied by the new measurement theory. We propose the following conjecture: All the twoorthogonal-settings experimental correlation functions admit local realistic theories irrespective of a state if we use the new measurement theory.

\section{Keywords}

Quantum Nonlocality, Quantum Measurement Theory

\section{Introduction}

As a famous physical theory, the quantum theory (cf. [1]-[5]) gives accurate and at times remarkably accurate numerical predictions. Much experimental data have fit to the quantum predictions for long time. 
On the other hand, from the incompleteness argument of Einstein, Podolsky, and Rosen (EPR) [6], a hiddenvariable interpretation of the quantum theory has been an attractive topic of research [2] [3]. There are two main approaches to study the hidden-variable interpretation of the quantum theory. One is the Bell-EPR theorem [7]. This theorem says that the quantum predictions violate the inequality following from the EPR-locality condition in the Hilbert space formalism of the quantum theory. The EPR-locality condition tells that a result of measurement pertaining to one system is independent of any measurement performed simultaneously at a distance on the other system.

The other is the no-hidden-variables theorem of Kochen and Specker (KS theorem) [8]. The original KS theorem says the non-existence of a real-valued function which is multiplicative and linear on commuting operators. Kochen and Specker constructed [8] a hidden-variable theory in two-dimensional space formalism of the quantum theory within von Neumann's projective measurement theory. In general, the quantum theory does not accept the KS type of hidden-variable theory. The proof of the original KS theorem relies on intricate geometric argument. Greenberger, Horne, and Zeilinger discovered [9] [10] the so-called GHZ theorem for four-partite GHZ state. And, the KS theorem becomes very simple form (see also Refs. [11]-[15]).

Mermin considers the Bell-EPR theorem in a multipartite state. He derives multipartite Bell inequality [16]. The quantum predictions by $n$-partite GHZ state violate the Bell-Mermin inequality by an amount that grows exponentially with $n$. And, several multipartite Bell inequalities are reported [17]-[25]. They also say that the quantum predictions violate local hidden-variable theories by an amount that grows exponentially with $n$.

As for the KS theorem, it is begun to research the validity of the KS theorem by using inequalities (see Refs. [26]-[29]). To find such inequalities to test the validity of the KS theorem is particularly useful for experimental investigation [30]. The KS theorem is related to the algebraic structure of a set of quantum operators. The KS theorem is independent of a quantum state under study. One of authors derives an inequality [29] as tests for the validity of the KS theorem. The quantum predictions violate the inequality when the system is in an uncorrelated state. An uncorrelated state is defined in Ref. [31]. The quantum predictions by $n$-partite uncorrelated state violate the inequality by an amount that grows exponentially with $n$.

Leggett-type nonlocal hidden-variable theory [32] is experimentally investigated [33]-[35]. The experiments report that the quantum theory does not accept Leggett-type nonlocal hidden-variable theory. These experiments are done in four-dimensional space (two parties) in order to study nonlocality of hidden-variable theories.

Recently, it is discussed that von Neumann's theory does not meet the Deutsch-Jozsa algorithm [36]. In von Neumann's theory, control of quantum state and observations of quantum state cannot be existential, simultaneously. In Ref. [36], we propose a solution of the problem. The problem is solved if measurement outcome is $\pm 1 / \sqrt{2}$.

Further, an additional condition for Bell experiments for accepting local realistic theories is discussed [37]. Again we can construct local realistic models if measurement outcome is $\pm 1 / \sqrt{2}$.

Therefore we consider the significance of the value $( \pm 1 / \sqrt{2})$ of the new measurement theory.

An important note here is that we use the original Bell-Mermin inequality. We want to investigate whether all Bell inequalities [23]-[25] with two-orthogonal-settings per side are satisfied by the new measurement theory even the GHZ state. If so, we can say that experimental measurement outcome $\pm 1 / \sqrt{2}$ is pre-determined. That is, the experimental correlation functions admit local realistic theories. As the first step, we investigate the original Bell-Mermin inequality. Our new measurement theory is equivalent to changing Planck's constant $(\hbar)$ to a new constant $(\hbar / \sqrt{2})$. We change the results of measurements instead of changing Planck's constant.

In this paper, we investigate the violation factor of the Bell-Mermin inequality. Until now, we have used an assumption that the results of measurement are \pm 1 . Recently, the new measurement theory is proposed [36]. The values of measurement outcome are $\pm 1 / \sqrt{2}$. Here we use the new measurement theory. We consider multipartite GHZ state. It turns out that the Bell-Mermin inequality is satisfied with the irrespective of the number of particles. We propose the following conjecture: All the two-orthogonal-settings experimental correlation functions admit local realistic theories irrespective of a state if we use the new measurement theory.

\section{Bell-Mermin Inequality Is Satisfied by the New Measurement Theory}

Let us consider $n$ particles $j=1,2, \cdots, n$. Let us consider $\sigma_{x}^{j}$ and $\sigma_{y}^{j}$ as Pauli observables for $j$ th particle. We insert $Q$ as an observable 


$$
Q=\operatorname{Re}\left(\prod_{j=1}^{n}\left(\sigma_{x}^{j}+i \sigma_{y}^{j}\right)\right)
$$

Let us consider the following GHZ state:

$$
\left|\Phi_{n}\right\rangle=\frac{1}{\sqrt{2}}\left(\left|+{ }_{1} ;+_{2} ; \cdots ;+_{n}\right\rangle+\left|-{ }_{1} ;-_{2} ; \cdots ;--_{n}\right\rangle\right) .
$$

We have the following experimental result

$$
\left\langle\Phi_{n}|Q| \Phi_{n}\right\rangle_{\text {experiment }}=2^{(n-1)} / \sqrt{2^{n}}=2^{(n-2) / 2}
$$

where the local results of measurements are $\pm 1 / \sqrt{2}$.

On the other hand, let us consider the original Bell-Mermin inequality. We consider $C$ as

$$
C=\operatorname{Re}\left(\prod_{j=1}^{n}\left(v\left(\sigma_{x}^{j}\right)+i v\left(\sigma_{y}^{j}\right)\right)\right)
$$

where $v\left(\sigma_{x}^{j}\right)= \pm 1$ and $v\left(\sigma_{y}^{j}\right)= \pm 1$. We see

$$
\begin{aligned}
& C \leq 2^{n / 2},(n=\text { even }), \\
& C \leq 2^{(n-1) / 2},(n=\text { odd }) .
\end{aligned}
$$

The maximum of $C$ is equal to the real part of a product of complex numbers each of which has magnitude of $\sqrt{2}$ and a phase of $\pm \pi / 4$ or $\pm 3 \pi / 4$. When $n$ is even the product can lie along the real axis and can attain a maximum value of $2^{n / 2}$, when $n$ is odd the product must lie along an axis at $\pm \pi / 4$ to the real axis and its real part can only attain the maximum value $2^{(n-1) / 2}$. Therefore, the value $C$ is bounded as (5).

Therefore, we have a violation of the Bell-Mermin inequality with the following factor

$$
\frac{\left\langle\Phi_{n}|Q| \Phi_{n}\right\rangle_{\text {experiment }}}{C}
$$

that is

$$
\begin{aligned}
& 1 / 2,(n=\text { even }), \\
& 1 / \sqrt{2},(n=\text { odd }) .
\end{aligned}
$$

We have

$$
\frac{\left\langle\Phi_{n}|Q| \Phi_{n}\right\rangle_{\text {experiment }}}{C}<1 .
$$

Hence, the Bell-Mermin inequality is satisfied irrespective of the number of particles. And the experimental correlation functions (measurement outcome is $\pm 1 / \sqrt{2}$ ) might admit local realistic theories.

\section{Conclusion}

In conclusion, we have investigated the violation factor of the Bell-Mermin inequality. Until now, we have used an assumption that the results of measurement are \pm 1 . Recently, a new measurement theory has been proposed. The values of measurement outcome have been $\pm 1 / \sqrt{2}$. Here we have used the new measurement theory. We have considered multipartite GHZ state. It has turned out that the Bell-Mermin inequality is satisfied irrespective of the number of particles. We have proposed the following conjecture: All the two-orthogonal-settings experimental correlation functions admit local realistic theories irrespective of a state if we use the new measurement theory.

\section{References}

[1] Sakurai, J.J. (1995) Modern Quantum Mechanics. Addison-Wesley Publishing Company, Revised Edition.

[2] Peres, A. (1993) Quantum Theory: Concepts and Methods. Kluwer Academic, Dordrecht. 
[3] Redhead, M. (1989) Incompleteness, Nonlocality, and Realism. 2nd Edition, Clarendon Press, Oxford.

[4] von Neumann, J. (1955) Mathematical Foundations of Quantum Mechanics. Princeton University Press, Princeton.

[5] Nielsen, M.A. and Chuang, I.L. (2000) Quantum Computation and Quantum Information. Cambridge University Press, Cambridge.

[6] Einstein, A., Podolsky, B. and Rosen, N. (1935) Can Quantum-Mechanical Description of Physical Reality Be Considered Complete? Physical Review, 47, 777. http://dx.doi.org/10.1103/PhysRev.47.777

[7] Bell, J.S. (1964) On the Einstein Podolsky Rosen Paradox. Physics, 1, 195-200.

[8] Kochen, S. and Specker, E.P. (1967) The Problem of Hidden Variables in Quantum Mechanics. Journal of Mathematics and Mechanics, 17, 59-87. http://dx.doi.org/10.1512/iumj.1968.17.17004

[9] Greenberger, D.M., Horne, M.A. and Zeilinger, A. (1989) Going Beyond Bell’s Theorem. In: Kafatos, M., Ed., Bell’s Theorem, Quantum Theory and Conceptions of the Universe, Kluwer Academic, Dordrecht, 69-72. http://dx.doi.org/10.1007/978-94-017-0849-4 10

[10] Greenberger, D.M., Horne, M.A., Shimony, A. and Zeilinger, A. (1990) Bell's Theorem without Inequalities. American Journal of Physics, 58, 1131-1143. http://dx.doi.org/10.1119/1.16243

[11] Pagonis, C., Redhead, M.L.G. and Clifton, R.K. (1991) The Breakdown of Quantum Non-Locality in the Classical Limit. Physics Letters A, 155, 441-444. http://dx.doi.org/10.1016/0375-9601(91)90643-M

[12] Mermin, N.D. (1990) What's Wrong with These Elements of Reality? Physics Today, 43, 9. http://dx.doi.org/10.1063/1.2810588

[13] Mermin, N.D. (1990) Quantum Mysteries Revisited. American Journal of Physics, 58, 731. http://dx.doi.org/10.1119/1.16503

[14] Peres, A. (1990) Incompatible Results of Quantum Measurements. Physics Letters A, 151, 107-108. http://dx.doi.org/10.1016/0375-9601(90)90172-K

[15] Mermin, N.D. (1990) Simple Unified Form for the Major No-Hidden-Variables Theorems. Physical Review Letters, 65, 3373. http://dx.doi.org/10.1103/PhysRevLett.65.3373

[16] Mermin, N.D. (1990) Extreme Quantum Entanglement in a Superposition of Macroscopically Distinct States. Physical Review Letters, 65, 1838. http://dx.doi.org/10.1103/PhysRevLett.65.1838

[17] Roy, S.M. and Singh, V. (1991) Tests of Signal Locality and Einstein-Bell Locality for Multiparticle Systems. Physical Review Letters, 67, 2761. http://dx.doi.org/10.1103/PhysRevLett.67.2761

[18] Ardehali, M. (1992) Bell Inequalities with a Magnitude of Violation That Grows Exponentially with the Number of Particles. Physical Review A, 46, 5375. http://dx.doi.org/10.1103/PhysRevA.46.5375

[19] Belinskii, A.V. and Klyshko, D.N. (1993) Interference of Light and Bell's Theorem. Physics-Uspekhi, 36, 653. http://dx.doi.org/10.1070/PU1993v036n08ABEH002299

[20] Werner, R.F. and Wolf, M.M. (2000) Bell’s Inequalities for States with Positive Partial Transpose. Physical Review A, 61, Article ID: 062102. http://dx.doi.org/10.1103/PhysRevA.61.062102

[21] Żukowski, M. (1993) Bell Theorem Involving All Settings of Measuring Apparatus. Physics Letters A, 177, $290-296$. http://dx.doi.org/10.1016/0375-9601(93)90002-H

[22] Żukowski, M. and Kaszlikowski, D. (1997) Critical Visibility for N-Particle Greenberger-Horne-Zeilinger Correlations to Violate Local Realism. Physical Review A, 56, R1682. http://dx.doi.org/10.1103/PhysRevA.56.R1682

[23] Żukowski, M. and Brukner, Č. (2002) Bell’s Theorem for General N-Qubit States. Physical Review Letters, 88, Article ID: 210401. http://dx.doi.org/10.1103/PhysRevLett.88.210401

[24] Werner, R.F. and Wolf, M.M. (2001) All-Multipartite Bell-Correlation Inequalities for Two Dichotomic Observables Per Site. Physical Review A, 64, Article ID: 032112. http://dx.doi.org/10.1103/PhysRevA.64.032112

[25] Werner, R.F. and Wolf, M.M. (2001) Bell Inequalities and Entanglement. Quantum Information \& Computation, 1, $1-25$.

[26] Simon, C., Brukner, Č. and Zeilinger, A. (2001) Hidden-Variable Theorems for Real Experiments. Physical Review Letters, 86, 4427. http://dx.doi.org/10.1103/PhysRevLett.86.4427

[27] Larsson, J.-Å. (2002) A Kochen-Specker Inequality. Europhysics Letters, 58, 799. http://dx.doi.org/10.1209/epl/i2002-00444-0

[28] Cabello, A. (2002) Finite-Precision Measurement Does Not Nullify the Kochen-Specker Theorem. Physical Review A, 65, Article ID: 052101. http://dx.doi.org/10.1103/PhysRevA.65.052101

[29] Nagata, K. and Math. J. (2005) Inequalities for Experimental Tests of the Kochen-Specker Theorem. Journal of Mathematical Physics, 46, Article ID: 102101. http://dx.doi.org/10.1063/1.2081115 
[30] Huang, Y.F., Li, C.F., Zhang, Y.S., Pan, J.W. and Guo, G.C. (2003) Experimental Test of the Kochen-Specker Theorem with Single Photons. Physical Review Letters, 90, Article ID: 250401. http://dx.doi.org/10.1103/PhysRevLett.90.250401

[31] Werner, R.F. (1989) Quantum States with Einstein-Podolsky-Rosen Correlations Admitting a Hidden-Variable Model. Physical Review A, 40, 4277. http://dx.doi.org/10.1103/PhysRevA.40.4277

[32] Leggett, A.J. (2003) Nonlocal Hidden-Variable Theories and Quantum Mechanics: An Incompatibility Theorem. Foundations of Physics, 33, 1469-1493. http://dx.doi.org/10.1023/A:1026096313729

[33] Gröblacher, S., Paterek, T., Kaltenbaek, R., Brukner, Č., Żukowski, M., Aspelmeyer, M. and Zeilinger, A. (2007) An Experimental Test of Non-Local Realism. Nature, 446, 871-875. http://dx.doi.org/10.1038/nature05677

[34] Paterek, T., Fedrizzi, A., Gröblacher, S., Jennewein, T., Żukowski, M., Aspelmeyer, M. and Zeilinger, A. (2007) Experimental Test of Nonlocal Realistic Theories without the Rotational Symmetry Assumption. Physical Review Letters, 99, Article ID: 210406. http://dx.doi.org/10.1103/PhysRevLett.99.210406

[35] Branciard, C., Ling, A., Gisin, N., Kurtsiefer, C., Lamas-Linares, A. and Scarani, V. (2007) Experimental Falsification of Leggett’s Nonlocal Variable Model. Physical Review Letters, 99, Article ID: 210407. http://dx.doi.org/10.1103/PhysRevLett.99.210407

[36] Nagata, K. and Nakamura, T. (2010) Can von Neumann’s Theory Meet the Deutsch-Jozsa Algorithm? International Journal of Theoretical Physics, 49, 162-170. http://dx.doi.org/10.1007/s10773-009-0189-5

[37] Nagata, K. and Nakamura, T. (2013) An Additional Condition for Bell Experiments for Accepting Local Realistic Theories. Quantum Information Processing, 12, 3785-3789. http://dx.doi.org/10.1007/s11128-013-0635-4 